\title{
ORGANOCHLORINES IN CHRYSICHTHYS NIGRODIGITATUS AND LIZA GRANDISQUAMIS FROM LAGOS LAGOON AND AGBOYI CREEK, NIGERIA
}

\author{
Akan B. Williams $^{1^{*}}$ and Olusegun O. Ayejuyo ${ }^{2}$ \\ ${ }^{1}$ Department of Chemistry, Covenant University, Ota, Nigeria \\ ${ }^{2}$ Department of Chemistry, University of Lagos, Lagos, Nigeria
}

(Received December 4, 2012; revised November 10, 2014)

\begin{abstract}
Catfish (Chrysichthys nigrodigitatus) and mullet (Liza grandisquamis) were sampled from Lagos Lagoon and Agboyi Creek and analysed for alpha-BHC, beta-BHC, lindane, delta-BHC, heptachlor, heptachlorepoxide (B), aldrin, dieldrin, endrin, endrin aldehyde, endrin ketone, cis-chlordane, trans-chlordane, endosulfan 1, endosulfan 11, endosulfan sulphate, methoxychlor, $p, p^{\prime}-D D E, p, p^{\prime}-D D D$ and p, $p^{\prime}$-DDT. Sampling was conducted four times at the designated sites during the dry season months of December 2008 and February 2009 and the wet season months of May and September 2009. The muscle tissues and organs of the finfishes were subjected to cold extraction with petroleum ether/acetone $(1: 1 \mathrm{v} / \mathrm{v})$ mixture and clean-up on silica gel adsorbents. A gas chromatograph coupled with electron capture detector (GC-ECD) was used to detect and determine the organochlorines. The residue levels of the fishes were higher in the Lagos Lagoon than in Agboyi Creek while a higher concentration of the residues was observed during the dry season. The female fishes accumulated higher organochlorines than the male fishes. The order of residue accumulation in the organs of the fishes was gills $>$ livers $>$ large intestines $>$ small intestines $>$ kidneys $>$ muscles. The dietary surveys indicated that the amount of fishes consumed ranged from 20 to $200 \mathrm{~g}$ /day, with a mean value of $40 \mathrm{~g} /$ day. The estimated daily intakes of the organochlorines were within the acceptable daily intakes while the levels of residues in the fishes were within the permissible residue limits.
\end{abstract}

KEY WORDS: Organochlorines, Lagos Lagoon, Agboyi Creek, Chrysichthys nigrodigitatus, Liza grandisquamis

\section{INTRODUCTION}

Nigeria is a rich fishery resource and fishes are major sources of proteins in the country. Finfishes constitute the major components of most aquatic habitats and are important biomonitors of residue levels in aquatic ecosystems. Fishes are suitable indicators for environmental pollution monitoring because they concentrate pollutants in their tissues directly from water and through their diet, thus enabling the assessment and transfer of pollutants through the trophic web $[1,2]$. The low activity of the mono-oxygenase enzymes in fishes limits their ability to metabolize organochlorines [3]. Hence, fishes reflect the levels of organochlorine pollution in the aquatic environment [4]. Besides evaluating organochlorines in the muscle tissues of fishes, investigating their distribution in organs of fishes is vital in order to provide more information about the pathways along which bioaccumulation occurs, and thus reflecting the environmental conditions.

Ingestion is the main source of human exposure to chlorinated pollutants [5]. Organochlorines are toxic environmental contaminants that are of concern [6]. They are highly toxic and bioaccumulate in the fatty tissues of animals and humans [7]. Biological monitoring of persistent organic pollutants provides information on the fate processes that the contaminants undergo [8]. Many persistent organic pollutants, become incorporated into food chain and consequently affect humans. Organochlorines could cause human breast and liver cancers, testicular tumours and lower sperm counts $[9,10]$.

Lagoons are depositories of effluent discharges, leachates and run-offs from activities on land. The discharge of rivers into lagoons is the main transport pathway of pesticide residues. The Nigerian coastal belt has estuaries and lagoons as a transition zone between it and the

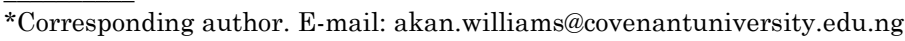


numerous rivers and creeks flowing southwards into the Atlantic Ocean. The distribution of various chlorinated hydrocarbons in the marine and estuarine environment depends on residues of persistent organochlorine pesticide physicochemical properties of the eco-system as well as partition coefficients of individual chlorinated hydrocarbons [11]. Organochlorines could distribute among the components of the ecosystem, such as water and sediment, and accumulate in aquatic organisms. The proliferation of urban settlements and slums in Lagos has led to increased human pressure and the generation of domestic effluents, which eventually find their way into the Lagos Lagoon. The lagoon therefore receives a complex mixture of domestic and industrial wastes and has served as the ultimate sink for the disposal of sewage. In view of the negative health effects of organochlorines on humans, it is necessary to evaluate the levels of these pollutants in the muscle tissues and organs of edible fishes. This study was therefore undertaken to investigate the levels of organochlorines in Chrysichthys nigrodigitatus and Liza grandisquamis, two fishes that are commonly consumed in Nigeria.

\section{EXPERIMENTAL}

\section{Study area}

The study area for the investigation is the Lagos Lagoon and Agboyi Creek. Lagos Lagoon lies between latitude $6^{\circ} 26^{\prime}-6^{\circ} 37^{\prime} \mathrm{N}$ and longitude $3^{\circ} 23^{\prime}-4^{\circ} 20^{\prime} \mathrm{E}$ while Agboyi Creek lies around latitude $6^{\circ} 33^{\prime}$ and longitude $3^{\circ} 25^{\prime}$ on the Western part of Nigeria. The lagoon consists of three main segments namely Lagos Harbour, Metropolitan End and Epe Division. It empties into the Atlantic Ocean via Lagos Harbour and is drained by Ogun, Agboyi, Majidun and Aye Rivers. Human activities associated with the lagoon include fishing, aquaculture and sand mining. The Agboyi Creek is one of the water bodies that drain the Lagos Lagoon. Organochlorine pesticides are still being used in Nigeria even though there is a paucity of data on their use in the country.

\section{Sampling strategy}

Sampling was conducted four times at Lagos Lagoon and Agboyi Creek during the dry season months of December 2008 and February 2009 and the wet season months of May and September 2009.

\section{Collection of fish samples}

Male and female catfish (Chrysichthys nigrodigitatus) and mullet (Liza grandisquamis) were harvested with the aid of fishing net. The harvested fish samples were separately wrapped in aluminium foil, stored in ice-packed coolers and transferred to the laboratory where they were frozen, thawed, cleaned in distilled water and their scales sloughed off.

\section{Determination of sex}

Catfish and mullet exhibit sexual dimorphism. Their gonads were examined in order to determine their gender. Gonads are the reproductive organs of a fish and consist of testis in the males and ovaries in the females and lie in the body cavity. The fish samples were separated into males and females by naked eye examination of their gonads [12].

\section{Evaluation of percentage (\%) dry matter}

1.0-2.0 g of muscle tissue of each of the fresh fish samples was weighed and dried in an oven maintained at $105{ }^{0} \mathrm{C}$ for 8 hours. The dried fish samples were cooled in desiccators and weighed in analytical balance to constant weight and the percentage dry matter was calculated. 


\section{Calculation of condition factor (CF)}

The condition factor (CF) which describes the physiological condition of the fishes [13] was calculated according to the equation [14]:

$$
C F=\frac{W}{L^{3}} \times 100
$$

where, $W=$ the fish wet weight $(\mathrm{g})$ and $L=$ the fish total length $(\mathrm{cm})$.

\section{Determination of fat content}

$10 \mathrm{~g}$ of fish muscle tissue was mixed with $10 \mathrm{~g}$ of anhydrous $\mathrm{Na}_{2} \mathrm{SO}_{4}$ to remove any trace of water and grinded with mortar and pestle. Cold solvent extraction was carried out using $50 \mathrm{~mL}$ petroleum ether/acetone $(1: 1 \mathrm{v} / \mathrm{v})$ mixture in a reagent bottle. The mixture was well shaken and allowed to stand for $30 \mathrm{~min}$ and then filtered. After evaporating the solvent extracts with the aid of a rotary evaporator, the fat content of the muscle tissue was determined gravimetrically:

$$
\% \text { Fat }=\frac{\text { weight of fat }}{\text { weight of tissue }} \times 100
$$

Sample preparation

Muscle tissue was dissected with a dissecting set between the pectoral fin and vent of each fish. The livers, gills, kidneys and intestines of the fishes were also extracted using the cold extraction method [15]. The solvent extracts were concentrated to $1 \mathrm{~mL}$ using a rotary evaporator and kept for clean-up. Column chromatography was used to clean-up the extracts [16]. The glass separating column was packed with activated silica gel $(90 \%<45 \mu \mathrm{m})$ and washed down with $\mathrm{n}$-hexane to remove dirts. The extracts were demoisturized over $1 \mathrm{~g}$ of anhydrous granulated $\mathrm{Na}_{2} \mathrm{SO}_{4}$ and separated into two eluted fractions using mixtures of dichloromethane, hexane and acetonitrile as eluting solvents. For the first fraction, $30 \mathrm{~mL}$ of a dichloromethane/hexane (20/80) mixture was used, while $30 \mathrm{~mL}$ of a dichloromethane/hexane/acetonitrile (50/49.5/0.5) mixture was used for the second fraction to ensure that the polar acetonitrile eluted any remaining residues. The fractions were combined, concentrated to $1 \mathrm{~mL}$ using a rotary evaporator and subsequently analysed for organochlorines.

\section{Identification and determination of organochlorines by gas chromatography}

The cleaned-up extracts were dried and re-dissolved in $1.0 \mathrm{~mL}$ isooctane [17]. Organochlorine Pesticides II EPA Method 8081A was used for the analyses. The detection and determination of the residues were performed by injecting $1 \mu \mathrm{L}$ of the $1.0 \mathrm{~mL}$ purified extract into the injection port of a gas chromatograph with a ${ }^{63} \mathrm{Ni}$ electron capture detector (GC- $\mu$ ECD Agilent Technology 7890A) equipped with the ChemStation software. Identification of pesticide residues was accomplished using reference standards and relative retention time techniques, while the residues were determined by comparing the peak heights of the samples with the corresponding peak heights of the reference standards of known concentrations. The concentrations $(\mathrm{w} / \mathrm{w})$ of the pesticide residues were calculated by the gas chromatograph after inputting the weight of the samples. The operating conditions of the gas chromatograph are presented in Table 1.

\section{Quality assurance}

Standards were run to check for the column performance, peak height, resolution and the detection limit. The correlation coefficients of calibration curves of the pesticides were all higher than 0.998 . The quality assurance measures included cleaning procedures, recovery of 
spiked standards and monitoring of detector response. Blank runs were made for background correction and performance of the system. The stock solution of the organochlorine pesticide standards was purchased from Restek Corporation, USA. It contained $1000 \mathrm{mg} / \mathrm{L}$ in n-hexane and was serially diluted to obtain the desired concentrations of 10,20 and $40 \mathrm{mg} / \mathrm{L}$. The recovery of organochlorines was carried out in replicate and was determined by spiking the previously analysed samples with the pesticide standard. The recovery percentages were calculated from the chromatograms.

Table 1. Operating conditions of the gas chromatograph.

\begin{tabular}{|l|l|}
\hline Detector & Electron capture \\
\hline Column & DB-5 fused silica capillary column $(30 \mathrm{~m}$ length $\times$ \\
& $\begin{array}{l}0.32 \mathrm{~mm} \text { i.d. } \times 0.25 \mu \mathrm{m} \text { film thickness). } \\
\mathrm{HP}-55 \% \text { Phenyl methyl siloxan }\end{array}$ \\
\hline Carrier gas & Helium $(99.9992)$ flowing at $20 \mathrm{~mL} / \mathrm{min}$ \\
\hline Carrier gas pressure & $10.744 \mathrm{psi}$ \\
\hline Make-up gas & Nitrogen $(99.9995 \%)$ \\
\hline Injector temperature & $250{ }^{\circ} \mathrm{C}$ \\
\hline Injection & $1 \mu \mathrm{L} \mathrm{Splitless}$ \\
\hline Detector temperature & $300{ }^{0} \mathrm{C}$ \\
\hline Temperature programme & Initial temp. $50{ }^{0} \mathrm{C}$ at a rate of $25{ }^{0} \mathrm{C} / \mathrm{min}$ to $100{ }^{0} \mathrm{C}$ \\
& $($ held for $1 \mathrm{~min})$, then at a rate of $5{ }^{0} \mathrm{C} / \mathrm{min}$ to $300{ }^{0} \mathrm{C}$ \\
\hline
\end{tabular}

Estimation of daily intakes (EDI) of organochlorines by humans

The daily intake of organochlorines by humans was estimated based on questionnaires and interviews conducted in 100 families consisting of 90 adults and 10 children. The respondents were persons who eat the fishes harvested and were categorized into males and females. Information on preference of the fish species, age and weight of the respondents and frequency of consumption was collated in order to estimate the daily dietary intake of the fishes. The respondents were mainly adults and a few children who reside in Lagos. Their economic activities cut across fishing, farming, teaching, public service and business. The average per capita consumption was estimated and compared with the acceptable daily intake. The dietary intake of the organochlorines was calculated by multiplying concentrations measured in the muscle tissues of each fish by the per capita consumption. The estimated daily intake of the organochlorines was calculated using the equation:

$$
\mathrm{EDI}=\frac{F D C}{B W} \times \mathrm{CC}
$$

where, $F D C=$ fish daily consumption $(\mathrm{g}), \mathrm{CC}=$ contaminant concentration $(\mathrm{ng} / \mathrm{g}), \quad B W=$ body weight $(\mathrm{kg})$ and $E D I=$ estimated daily intake of OCPs ( $\mathrm{ng} / \mathrm{kg}$ body weight/day). $40 \mathrm{~g}$ was taken as the average fish daily consumption while $70 \mathrm{~kg}$ was taken as the average body weight.

\section{Analyses of data}

The fish extracts were analysed for alpha-BHC, beta-BHC, lindane, delta-BHC, heptachlor, heptachlor-epoxide (B), aldrin, dieldrin, endrin, endrin aldehyde, endrin ketone, cis-chlordane, trans-chlordane, endosulfan 1, endosulfan 11, endosulfan sulphate, methoxychlor, p,p'-DDE, $\mathrm{p}, \mathrm{p}^{\prime}$-DDD and $\mathrm{p}, \mathrm{p}^{\prime}$-DDT. Concentrations of OCP residues were calculated individually and as the sum of their isomeric forms. Description of data was performed using a Statgraphics Centurion XV statistical software, with the level of significance maintained at $95 \%$ for each test. 
The mean and standard deviation were calculated from the detectable values, and values below the detectable limit were considered not detected (ND). The mean was calculated from triplicate determinations.

\section{RESULTS AND DISCUSSION}

The mean biometric data of Chrysichthys nigrodigitatus and Liza grandisquamis are presented in Tables 2-3. There was a positive correlation between the total lengths and standard lengths of the fishes. Increasing fish lengths gave corresponding increases in fish weights. There was no correlation between the wet weights and \% fat of the fish species. The fishes had condition factor less than 1 during the dry season. The condition factor describes the physiological condition of fishes [13] and usually increases when sexual maturation approaches. An undernourished or thin fish has a condition factor less than 1 while an adequately fed or fat fish has a condition factor greater than 1 . The condition factor could be variable between samples due to anatomical differences. The condition factor of the fishes was generally low, giving an indication of their physiological state. The condition factor of the fishes in Agboyi creek was higher than those from the lagoon mainly due to the higher level of pollution in the Lagos Lagoon, feeding mode and availability of food for the fishes. The percentage of recovery of the residues ranged between $88.5 \pm 2.8$ and $98.4 \pm 2.8 \%$.

Table 2. Mean biometric data of Chrysichthys nigrodigitatus and Liza grandisquamis in Agboyi Creek during the dry and wet seasons.

\begin{tabular}{|c|c|c|c|c|c|c|c|c|}
\hline Fish species & $\begin{array}{c}\text { Feeding } \\
\text { mode }\end{array}$ & Sex & $\begin{array}{c}\text { Wet } \\
\text { weight }(\mathrm{g})\end{array}$ & $\begin{array}{c}\text { \% Dry } \\
\text { matter }\end{array}$ & $\begin{array}{c}\text { \% Fat (fish } \\
\text { muscle) }\end{array}$ & $\begin{array}{c}\text { TL } \\
(\mathrm{cm})\end{array}$ & $\begin{array}{c}\text { SL } \\
(\mathrm{cm})\end{array}$ & CF \\
\hline \multicolumn{8}{|c|}{ Dry Season } \\
\hline \multirow{2}{*}{ C. nigrodigitatus } & Omnivorous & Male & $75.3 \pm 0.4$ & $23.2 \pm 0.3$ & $0.8 \pm 0.1$ & $23.0 \pm 0.4$ & $17.0 \pm 0.4$ & $0.6 \pm 0.3$ \\
\cline { 3 - 9 } & & Female & $110 \pm 0.1$ & $25.4 \pm 0.9$ & $0.9 \pm 0.1$ & $23.0 \pm 0.8$ & $18.5 \pm 0.8$ & $0.9 \pm 0.6$ \\
\hline L. grandisquamis & Omnivorous & Male & $48.0 \pm 0.1$ & $19.4 \pm 0.6$ & $0.2 \pm 0.1$ & $20.0 \pm 0.1$ & $15.0 \pm 0.1$ & $0.6 \pm 0.1$ \\
\cline { 3 - 8 } & Female & $45.0 \pm 0.2$ & $19.2 \pm 0.4$ & $0.2 \pm 0.2$ & $20.0 \pm 0.2$ & $15.0 \pm 0.2$ & $0.6 \pm 0.2$ \\
\hline \multicolumn{8}{|c|}{ Wet Season } \\
\hline C. nigrodigitatus & Omnivorous & Male & $74.2 \pm 0.7$ & $23.1 \pm 0.1$ & $0.8 \pm 0.1$ & $23.0 \pm 0.6$ & $17.0 \pm 0.6$ & $1.5 \pm 0.5$ \\
\cline { 3 - 9 } & Female & $90.1 \pm 0.1$ & $24.6 \pm 0.8$ & $0.8 \pm 0.1$ & $23.0 \pm 0.1$ & $18.5 \pm 0.9$ & $1.4 \pm 0.8$ \\
\hline \multirow{2}{*}{ L. grandisquamis } & Omnivorous & Male & $48.3 \pm 0.4$ & $19.0 \pm 0.5$ & $0.2 \pm 0.2$ & $20.0 \pm 0.4$ & $15.0 \pm 0.4$ & $0.6 \pm 0.4$ \\
\cline { 3 - 9 } & Female & $45.4 \pm 0.6$ & $18.9 \pm 0.6$ & $0.2 \pm 0.3$ & $20.0 \pm 0.5$ & $15.0 \pm 0.5$ & $0.6 \pm 0.4$ \\
\hline
\end{tabular}

$\mathrm{TL}=$ total length of wet fish; $\mathrm{SL}=$ standard length of wet fish; $\mathrm{CF}=$ condition factor of fish. The mean value was calculated from 3 fishes of each species.

Table 3. Mean biometric data of Chrysichthys nigrodigitatus and Liza grandisquamis in Lagos Lagoon during the dry and wet seasons.

\begin{tabular}{|c|c|c|c|c|c|c|c|c|}
\hline Fish species & $\begin{array}{c}\text { Feeding } \\
\text { mode }\end{array}$ & Sex & $\begin{array}{c}\text { Wet } \\
\text { weight (g) }\end{array}$ & $\begin{array}{c}\text { \% Dry } \\
\text { matter }\end{array}$ & $\begin{array}{c}\text { \% Fat (fish } \\
\text { muscle) }\end{array}$ & $\begin{array}{c}\text { TL } \\
(\mathrm{cm})\end{array}$ & $\begin{array}{c}\text { SL } \\
(\mathrm{cm})\end{array}$ & CF \\
\hline \multicolumn{8}{|c|}{ Dry Season } \\
\hline C. nigrodigitatus & Omnivorous & Male & $52.3 \pm 0.2$ & $28.8 \pm 0.2$ & $0.5 \pm 0.1$ & $20.0 \pm 0.2$ & $16.0 \pm 0.2$ & $0.7 \pm 0.2$ \\
\cline { 3 - 9 } & & Female & $55.2 \pm 0.4$ & $29.3 \pm 0.4$ & $0.6 \pm 0.1$ & $20.5 \pm 0.4$ & $16.5 \pm 0.3$ & $0.6 \pm 0.3$ \\
\hline \multirow{2}{*}{ L. grandisquamis } & Omnivorous & Male & $134 \pm 0.8$ & $38.4 \pm 0.7$ & $0.4 \pm 0.1$ & $24.5 \pm 0.7$ & $20.0 \pm 0.6$ & $0.9 \pm 0.2$ \\
\cline { 3 - 9 } & Female & $108 \pm 0.1$ & $35.4 \pm 0.8$ & $0.3 \pm 0.2$ & $25.0 \pm 0.7$ & $20.0 \pm 0.8$ & $0.7 \pm 0.1$ \\
\hline \multicolumn{8}{|c|}{ Wet Season } \\
\hline C. nigrodigitatus & Omnivorous & Male & $50.3 \pm 0.3$ & $28.5 \pm 0.3$ & $0.5 \pm 0.2$ & $20.0 \pm 0.3$ & $16.0 \pm 0.3$ & $0.6 \pm 0.3$ \\
\cline { 3 - 9 } & Female & $53.2 \pm 0.5$ & $29.0 \pm 0.5$ & $0.6 \pm 0.2$ & $20.8 \pm 0.5$ & $16.8 \pm 0.4$ & $0.6 \pm 0.2$ \\
\hline \multirow{2}{*}{ L. grandisquamis } & Omnivorous & Male & $168 \pm 0.8$ & $18.9 \pm 0.6$ & $0.4 \pm 0.1$ & $24.5 \pm 0.7$ & $20.0 \pm 0.7$ & $1.1 \pm 0.4$ \\
\cline { 3 - 8 } & Female & $126 \pm 0.5$ & $35.0 \pm 0.4$ & $0.3 \pm 0.1$ & $30.0 \pm 0.5$ & $25.0 \pm 0.4$ & $0.5 \pm 0.4$ \\
\hline
\end{tabular}




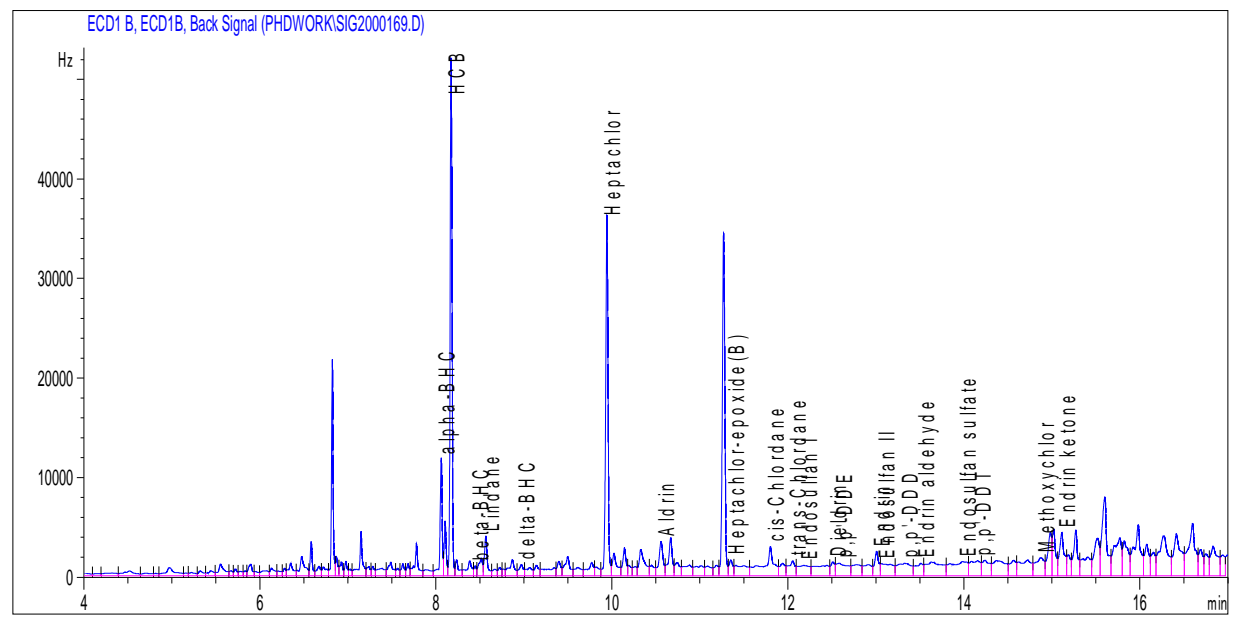

Figure 1. Chromatogram of organochlorine pesticide residues in the liver of male Chrysichthys nigrodigitatus during the dry season in Lagos Lagoon.

A typical chromatogram of organochlorine pesticide residues in the liver of male Chrysichthys nigrodigitatus during the dry season in Lagos Lagoon is shown in Figure 1. The mean concentrations of organochlorines in muscle tissues and organs of the male and female fishes are shown in Tables 4-12. The level of organochlorine residues in the fishes in Lagos Lagoon was higher than the level in Agboyi Creek while a higher concentration of the residues was observed during the dry season. This is as a result of the higher contamination of the Lagos Lagoon and dilution effect that is witnessed during the wet season. The muscle tissues of the female fishes accumulated higher organochlorines than the muscle tissues of the male fishes, possibly due to physiological differences such as increased fat content. There was no consistent pattern in the residue accumulation by male and female organs of the fish species studied. The dominant BHC was beta-BHC. BHCs accumulation largely followed the order beta-BHC > lindane $>$ delta-BHC $>$ alpha-BHC. The total dichlorodiphenyltrichloroethane (DDT) concentration in this study followed the order: $p, p^{\prime}$-DDT $>$ p, $\mathrm{p}^{\prime}$-DDD $>\mathrm{p}, \mathrm{p}^{\prime}$-DDE. The high $\mathrm{p}, \mathrm{p}^{\prime}$-DDT levels detected in this study was in contrast with previous studies [19] which showed that $\mathrm{p}, \mathrm{p}^{\prime}-\mathrm{DDE}$ was the major DDT residue in aquatic species. The higher proportion of DDT than its metabolites indicates that some of the contamination could be recent. In the environment, DDT breaks down into its major metabolites, dichlorodiphenylethane (DDE) and dichlorodiphenyldichloroethane (DDD) over time. The continued use of DDT in some countries, including Nigeria, further contributes to worldwide environmental contamination. DDE has the shortest biological half-life, followed by DDT and then DDD. It is the persistence of DDT and its breakdown products that leads to its bioaccumulation and bio-concentration in the food chain. The fat solubility of DDT results in its being concentrated throughout the food chain.

In this study, the total detectable organochlorines (wet weight) of the muscle tissues ranged between $3.8 \mathrm{ng} / \mathrm{g}$ in C. nigrodigitatus and $2082 \mathrm{ng} / \mathrm{g}$ in L. grandisquamis and were higher when compared to earlier studies in Ogun and Edo Rivers [20, 21]. However, the levels of detectable organochlorines were lower when compared to studies conducted by Adeyemi et al. [22] in Lagos Lagoon. 
Table 4. Mean concentrations (ng/g) of organochlorines in the muscle tissues of male and female Chrysichthys nigrodigitatus during the dry and wet seasons.

\begin{tabular}{|c|c|c|c|c|c|c|c|c|}
\hline \multirow[b]{3}{*}{ OCPs } & \multicolumn{4}{|c|}{ Agboyi Creek } & \multicolumn{4}{|c|}{ Lagos Lagoon } \\
\hline & \multicolumn{2}{|c|}{ Dry season } & \multicolumn{2}{|c|}{ Wet season } & \multicolumn{2}{|c|}{ Dry season } & \multicolumn{2}{|c|}{ Wet season } \\
\hline & Male & Female & Male & Female & Male & Female & Male & Female \\
\hline Alpha-BHC & $53.1 \pm 2.3$ & $10.6 \pm 2.5$ & $1.4 \pm 0.2$ & $1.5 \pm 0.4$ & $59.6 \pm 1.6$ & $14.2 \pm 2.3$ & ND & ND \\
\hline Beta-BHC & $54.9 \pm 3.2$ & $67.1 \pm 2.2$ & $2.1 \pm 0.3$ & $2.2 \pm 1.7$ & $16.9 \pm 2.1$ & $54.1 \pm 3.0$ & $0.3 \pm 0.4$ & $0.2 \pm 0.8$ \\
\hline Lindane & $10.7 \pm 4.3$ & $27.8 \pm 3.3$ & $1.2 \pm 1.0$ & $1.3 \pm 0.4$ & $5.4 \pm 2.8$ & $27.5 \pm 2.6$ & $0.6 \pm 0.1$ & $0.6 \pm 0.3$ \\
\hline Delta-BHC & $10.4 \pm 2.1$ & $28.5 \pm 5.2$ & $0.9 \pm 0.3$ & $0.9 \pm 0.2$ & $8.8 \pm 3.0$ & $12.5 \pm 1.1$ & ND & $0.7 \pm 0.2$ \\
\hline$\Sigma \mathrm{BHC}$ & $129 \pm 12$ & $134 \pm 13$ & $5.6 \pm 1.8$ & $5.9 \pm 2.7$ & $90.7 \pm 9.5$ & $108 \pm 9.0$ & $0.9 \pm 0.5$ & $1.4 \pm 1.3$ \\
\hline Heptachlor & $64.0 \pm 5.1$ & $7.7 \pm 1.2$ & $1.0 \pm 0.4$ & $1.0 \pm 1.1$ & $254 \pm 9.4$ & $14.3 \pm 2.0$ & $0.8 \pm 0.2$ & $0.9 \pm 0.2$ \\
\hline $\begin{array}{l}\text { Heptachlor } \\
\text {-epoxide (B) }\end{array}$ & $5.1 \pm 2.5$ & $10.8 \pm 3.3$ & $0.5 \pm 0.5$ & $0.6 \pm 0.2$ & $11.9 \pm 3.5$ & $38.3 \pm 2.1$ & ND & ND \\
\hline Aldrin & $22.5 \pm 4.3$ & $24.6 \pm 2.4$ & $0.7 \pm 0.2$ & $0.8 \pm 0.3$ & $30.1 \pm 5.7$ & $12.9 \pm 1.9$ & $0.9 \pm 0.3$ & $1.2 \pm 0.2$ \\
\hline Dieldrin & ND & $3.8 \pm 1.5$ & ND & ND & $9.8 \pm 1.5$ & $57.9 \pm 2.4$ & ND & ND \\
\hline Endrin & $37.5 \pm 4.5$ & $18.1 \pm 2.2$ & $6.9 \pm 2.0$ & $7.6 \pm 2.0$ & $20.1 \pm 2.7$ & $82.1 \pm 3.0$ & $0.4 \pm 0.5$ & $0.5 \pm 0.4$ \\
\hline $\begin{array}{l}\text { Endrin } \\
\text { aldehyde }\end{array}$ & $20.6 \pm 5.3$ & ND & ND & ND & $38.9 \pm 5.9$ & $279 \pm 6.0$ & ND & ND \\
\hline Endrin ketone & ND & $15.5 \pm 2.4$ & $225 \pm 0.4$ & $238 \pm 8.4$ & $123 \pm 2.3$ & ND & ND & ND \\
\hline Cis-Chlordane & $21.3 \pm 1.5$ & $13.3 \pm 3.6$ & $0.6 \pm 0.3$ & $0.6 \pm 1.1$ & $23.5 \pm 3.6$ & $4.1 \pm 2.0$ & ND & ND \\
\hline $\begin{array}{l}\text { Trans- } \\
\text { Chlordane } \\
\end{array}$ & $9.9 \pm 2.2$ & $19.7 \pm 5.1$ & ND & ND & $20.2 \pm 4.3$ & $27.3 \pm 7.0$ & ND & ND \\
\hline Endosulfan 1 & $5.6 \pm 2.3$ & $19.6 \pm 4.1$ & $0.9 \pm 0.4$ & $0.9 \pm 0.5$ & $10.6 \pm 2.5$ & $20.4 \pm 4.1$ & $0.6 \pm 0.6$ & $0.7 \pm 0.3$ \\
\hline Endosulfan 11 & $7.9 \pm 1.5$ & $7.9 \pm 2.2$ & ND & ND & $7.4 \pm 1.6$ & $54.0 \pm 3.0$ & ND & ND \\
\hline $\begin{array}{l}\text { Endosulfan } \\
\text { sulphate }\end{array}$ & ND & ND & ND & ND & $26.4 \pm 3.4$ & $227 \pm 6.0$ & ND & ND \\
\hline Methoxychlor & ND & 0 & ND & ND & $9.2 \pm 6.8$ & $314 \pm 3.0$ & ND & ND \\
\hline $\mathrm{p}, \mathrm{p}^{\prime}-\mathrm{DDE}$ & $26.8 \pm 2.3$ & $3.9 \pm 1.3$ & ND & ND & $4.9 \pm 2.2$ & $111 \pm 2.0$ & ND & ND \\
\hline p,p'-DDD & $6.0 \pm 1.2$ & $9.3 \pm 3.5$ & $8.8 \pm 2.2$ & $9.7 \pm 3.3$ & $6.6 \pm 1.0$ & $199 \pm 3.0$ & ND & ND \\
\hline $\mathrm{p}, \mathrm{p}^{\prime}-\mathrm{DDT}$ & $16.2 \pm 4.4$ & ND & ND & ND & $22.3 \pm 2.0$ & $472 \pm 5.1$ & ND & ND \\
\hline$\Sigma \mathrm{DDT}$ & $49.0 \pm 7.9$ & $13.2 \pm 4.8$ & $8.8 \pm 2.2$ & $9.7 \pm 3.3$ & $33.8 \pm 5.2$ & $781 \pm 10.1$ & ND & ND \\
\hline$\Sigma \mathrm{OCPs}$ & $373 \pm 49$ & $288 \pm 46$ & $250 \pm 8.2$ & $266 \pm 20$ & $910 \pm 68$ & $2041 \pm 62$ & $3.8 \pm 2.1$ & $4.6 \pm 2.4$ \\
\hline
\end{tabular}

Table 5. Mean concentrations (ng/g) of organochlorines in organs of male and female Chrysichthys nigrodigitatus during the dry season in Agboyi Creek.

\begin{tabular}{|l|c|c|c|c|c|c|}
\hline \multirow{2}{*}{ OCPs } & \multicolumn{5}{|c|}{ Agboyi Creek } & \multicolumn{2}{c|}{ Gill } \\
\cline { 2 - 7 } & \multicolumn{2}{|c|}{ Liver } & \multicolumn{2}{c|}{ Kidney } & Male & Female \\
\cline { 2 - 7 } Alpha-BHC & $57.6 \pm 8.6$ & $62.5 \pm 5.2$ & $36.8 \pm 8.7$ & $38.6 \pm 7.9$ & $15.2 \pm 8.2$ & $18.5 \pm 6.3$ \\
\hline Beta-BHC & $75.3 \pm 6.5$ & $87.5 \pm 7.9$ & $65.3 \pm 5.2$ & $68.9 \pm 8.7$ & $80.2 \pm 9.6$ & $92.9 \pm 9.0$ \\
\hline Lindane & $29.6 \pm 8.1$ & $33.2 \pm 4.3$ & $138 \pm 54$ & $150 \pm 64$ & $30.7 \pm 6.3$ & $32.4 \pm 8.9$ \\
\hline Delta-BHC & $34.8 \pm 11.5$ & $36.5 \pm 6.3$ & $51.9 \pm 6.4$ & $60.4 \pm 9.2$ & $86.5 \pm 9.0$ & $95.3 \pm 9.5$ \\
\hline SBHC & $197 \pm 35$ & $220 \pm 24$ & $292 \pm 74$ & $317 \pm 32$ & $213 \pm 33$ & $239 \pm 34$ \\
\hline Heptachlor & $12.9 \pm 8.3$ & $15.7 \pm 5.2$ & $38.1 \pm 9.2$ & $43.7 \pm 8.0$ & $49.6 \pm 8.8$ & $52.8 \pm 8.4$ \\
\hline $\begin{array}{l}\text { Heptachlor } \\
\text {-epoxide (B) }\end{array}$ & $18.8 \pm 8.1$ & $21.4 \pm 4.1$ & $134 \pm 5.7$ & $155 \pm 8.2$ & $202 \pm 5.2$ & $215 \pm 12$ \\
\hline Aldrin & $30.2 \pm 6.7$ & $35.8 \pm 9.2$ & $81.8 \pm 11.5$ & $87.5 \pm 6.2$ & $127 \pm 8.9$ & $141 \pm 7.4$ \\
\hline Dieldrin & $8.1 \pm 3.5$ & $14.8 \pm 12.1$ & $97.4 \pm 8.4$ & $98.1 \pm 6.2$ & $120 \pm 7.7$ & $136 \pm 9.3$ \\
\hline Endrin & $24.8 \pm 8.2$ & $28.2 \pm 9.2$ & $236 \pm 4.8$ & $257 \pm 8.7$ & $297 \pm 24$ & $312 \pm 8.1$ \\
\hline Endrin aldehyde & $32.9 \pm 7.6$ & $38.7 \pm 7.4$ & $404 \pm 9.6$ & $429 \pm 9.4$ & $686 \pm 9.3$ & $703 \pm 6.1$ \\
\hline Endrin ketone & $25.5 \pm 6.2$ & $28.3 \pm 5.7$ & $548 \pm 5.7$ & $565 \pm 6.1$ & $515 \pm 7.0$ & $539 \pm 8.2$ \\
\hline Cis-Chlordane & $21.6 \pm 4.2$ & $27.7 \pm 9.3$ & $135 \pm 8.5$ & $151 \pm 8.6$ & $264 \pm 9.7$ & $273 \pm 9.2$ \\
\hline Trans-Chlordane & $28.7 \pm 9.4$ & $37.8 \pm 8.1$ & $66.6 \pm 8.8$ & $87.5 \pm 4.2$ & $170 \pm 12$ & $171 \pm 6.5$ \\
\hline Endosulfan 1 & $35.2 \pm 4.2$ & $39.0 \pm 7.8$ & $107 \pm 5.3$ & $123 \pm 4.3$ & $143 \pm 8.8$ & $150 \pm 5.3$ \\
\hline Endosulfan 11 & $12.6 \pm 4.4$ & $30.5 \pm 10.9$ & $90.3 \pm 4.3$ & $98.2 \pm 8.9$ & $\mathrm{ND}$ & $\mathrm{ND}$ \\
\hline Endosulfan & $\mathrm{ND}$ & $\mathrm{ND}$ & $313 \pm 7.9$ & $333 \pm 7.3$ & $605 \pm 9.1$ & $619 \pm 7.4$ \\
\hline
\end{tabular}

Bull. Chem. Soc. Ethiop. 2015, 29(2) 


\begin{tabular}{|l|c|c|c|c|c|c|}
\hline sulphate & & & & & & \\
\hline Methoxychlor & $23.7 \pm 6.3$ & $28.4 \pm 9.4$ & $79.4 \pm 7.9$ & $91.2 \pm 4.2$ & $232 \pm 6.2$ & $239 \pm 9.3$ \\
\hline p,p'-DDE & $9.7 \pm 8.8$ & $12.3 \pm 7.1$ & $197 \pm 5.6$ & $206 \pm 6.9$ & $167 \pm 7.2$ & $183 \pm 8.8$ \\
\hline p,p'-DDD & $13.2 \pm 4.3$ & $18.8 \pm 8.2$ & $261 \pm 4.1$ & $278 \pm 8.3$ & ND & ND \\
\hline p,p'-DDT & $6.6 \pm 6.7$ & $9.3 \pm 9.8$ & $227 \pm 6.2$ & $243 \pm 7.9$ & $370 \pm 8.4$ & $385 \pm 13$ \\
\hline$\Sigma$ DDT & $29.4 \pm 19.9$ & $40.4 \pm 25.2$ & $685 \pm 16$ & $727 \pm 23$ & $538 \pm 16$ & $568 \pm 21$ \\
\hline$\Sigma$ OCPs & $501 \pm 132$ & $607 \pm 147$ & $3307 \pm 188$ & $3563 \pm 146$ & $4160 \pm 166$ & $4355 \pm 153$ \\
\hline
\end{tabular}

Table 6. Mean concentrations (ng/g) of organochlorines in organs of male and female Chrysichthys nigrodigitatus during the wet season in Agboyi Creek.

\begin{tabular}{|c|c|c|c|c|c|c|}
\hline \multirow[b]{3}{*}{ OCPs } & \multicolumn{6}{|c|}{ Agboyi Creek } \\
\hline & \multicolumn{2}{|c|}{ Liver } & \multicolumn{2}{|c|}{ Kidney } & \multicolumn{2}{|c|}{ Gill } \\
\hline & Male & Female & Male & Female & Male & Female \\
\hline Alpha-BHC & $24.7 \pm 9.1$ & $32.9 \pm 1.4$ & $26.1 \pm 2.1$ & $32.9 \pm 9.3$ & $45.6 \pm 6.8$ & $58.5 \pm 5.3$ \\
\hline Beta-BHC & $54.7 \pm 6.5$ & $61.8 \pm 2.1$ & $56.9 \pm 1.6$ & $62.4 \pm 7.2$ & $77.9 \pm 8.1$ & $86.5 \pm 6.8$ \\
\hline Lindane & $20.6 \pm 4.1$ & $38.3 \pm 1.7$ & $74.3 \pm 6.2$ & $98.6 \pm 4.7$ & $39.4 \pm 5.3$ & $38.1 \pm 7.2$ \\
\hline Delta-BHC & $37.6 \pm 6.3$ & $39.7 \pm 9.3$ & $45.1 \pm 5.9$ & $66.5 \pm 7.4$ & $76.8 \pm 8.2$ & $89.6 \pm 6.7$ \\
\hline$\Sigma \mathrm{BHC}$ & $138 \pm 26$ & $173 \pm 15$ & $202 \pm 15.9$ & $261 \pm 29$ & $240 \pm 28$ & $273 \pm 26$ \\
\hline Heptachlor & $10.5 \pm 7.8$ & $13.8 \pm 8.2$ & $38.0 \pm 7.3$ & $39.1 \pm 7.8$ & $36.2 \pm 2.2$ & $40.2 \pm 6.7$ \\
\hline $\begin{array}{l}\text { Heptachlor } \\
\text {-epoxide (B) }\end{array}$ & $13.8 \pm 7.8$ & $16.9 \pm 6.3$ & $105 \pm 5.3$ & $114 \pm 4.8$ & $187 \pm 1.7$ & $194 \pm 5.1$ \\
\hline Aldrin & $20.8 \pm 9.2$ & $25.8 \pm 5.3$ & $51.9 \pm 3.1$ & $54.3 \pm 9.1$ & $96.3 \pm 8.8$ & $106 \pm 8.4$ \\
\hline Dieldrin & $5.1 \pm 6.5$ & $7.6 \pm 6.2$ & $68.4 \pm 4.8$ & $65.8 \pm 6.1$ & $90.9 \pm 4.5$ & $108 \pm 4.8$ \\
\hline Endrin & $12.0 \pm 7.1$ & $19.7 \pm 9.2$ & $206 \pm 2.7$ & $219 \pm 7.3$ & $232 \pm 7.5$ & $267 \pm 6.8$ \\
\hline $\begin{array}{l}\text { Endrin } \\
\text { aldehyde }\end{array}$ & $21.9 \pm 8.4$ & $31.9 \pm 5.1$ & $365 \pm 7.6$ & $388 \pm 4.2$ & $615 \pm 4.3$ & $655 \pm 7.9$ \\
\hline Endrin ketone & $18.9 \pm 3.2$ & $19.4 \pm 8.6$ & $521 \pm 3.5$ & $518 \pm 8.7$ & $477 \pm 8.9$ & $510 \pm 3.3$ \\
\hline Cis-Chlordane & $13.6 \pm 2.9$ & $18.7 \pm 6.3$ & $105 \pm 8.3$ & $110 \pm 9.2$ & $209 \pm 3.5$ & $225 \pm 2.9$ \\
\hline $\begin{array}{l}\text { Trans- } \\
\text { Chlordane }\end{array}$ & $18.9 \pm 6.0$ & $23.2 \pm 8.5$ & $42.6 \pm 2.6$ & $56.2 \pm 8.8$ & $127 \pm 9.2$ & $145 \pm 7.3$ \\
\hline Endosulfan 1 & $27.6 \pm 8.3$ & $31.1 \pm 5.5$ & $96.5 \pm 8.1$ & $117 \pm 6.4$ & $123 \pm 2.9$ & $126 \pm 6.2$ \\
\hline Endosulfan 11 & $10.2 \pm 2.1$ & $24.2 \pm 9.6$ & $60.7 \pm 4.4$ & $82.6 \pm 9.4$ & ND & ND \\
\hline $\begin{array}{l}\text { Endosulfan } \\
\text { sulphate }\end{array}$ & ND & ND & $251 \pm 5.9$ & $281 \pm 8.3$ & $564 \pm 5.5$ & $575 \pm 8.2$ \\
\hline Methoxychlor & $20.9 \pm 1.2$ & $22.8 \pm 8.7$ & $52.1 \pm 3.2$ & $76.2 \pm 6.5$ & $211 \pm 3.3$ & $225 \pm 5.7$ \\
\hline $\mathrm{p}, \mathrm{p}^{\prime}-\mathrm{DDE}$ & $7.6 \pm 5.4$ & $10.4 \pm 6.1$ & $159 \pm 9.6$ & $187 \pm 8.2$ & $130 \pm 9.9$ & $165 \pm 8.4$ \\
\hline p,p'-DDD & $11.1 \pm 5.2$ & $13.9 \pm 5.6$ & $221 \pm 4.8$ & $219 \pm 6.4$ & ND & ND \\
\hline $\mathrm{p}, \mathrm{p}^{\prime}-\mathrm{DDT}$ & $4.8 \pm 7.0$ & $6.4 \pm 8.3$ & $203 \pm 8.3$ & $218 \pm 9.4$ & $321 \pm 6.9$ & $314 \pm 12$ \\
\hline$\Sigma$ DDT & $23.5 \pm 18$ & $30.8 \pm 20$ & $583 \pm 23$ & $624 \pm 24$ & $451 \pm 17$ & $479 \pm 21$ \\
\hline$\Sigma$ OCPs & $355 \pm 114$ & $459 \pm 122$ & $2748 \pm 108$ & $3005 \pm 149$ & $3660 \pm 108$ & $3928 \pm 120$ \\
\hline
\end{tabular}

Table 7. Mean concentrations (ng/g) of organochlorines in organs of male and female Chrysichthys nigrodigitatus during the dry season in Lagos Lagoon.

\begin{tabular}{|c|c|c|c|c|c|c|}
\hline \multirow[b]{3}{*}{ OCPs } & \multicolumn{6}{|c|}{ Lagos Lagoon } \\
\hline & \multicolumn{2}{|c|}{ Liver } & \multicolumn{2}{|c|}{ Kidney } & \multicolumn{2}{|c|}{ Gill } \\
\hline & Male & Female & Male & Female & Male & Female \\
\hline Alpha-BHC & $54.3 \pm 8.2$ & $58.5 \pm 4.2$ & $33.4 \pm 3.9$ & $38.9 \pm 7.5$ & $278 \pm 8.3$ & $293 \pm 5.2$ \\
\hline Beta-BHC & $15.1 \pm 1.1$ & $20.9 \pm 2.6$ & $2.9 \pm 3.2$ & $4.6 \pm 5.0$ & $634 \pm 7.1$ & $686 \pm 8.3$ \\
\hline Lindane & $28.8 \pm 2.8$ & $32.9 \pm 9.4$ & $6.1 \pm 7.0$ & $10.5 \pm 3.2$ & $108 \pm 4.1$ & $126 \pm 5.9$ \\
\hline Delta-BHC & $14.6 \pm 4.1$ & $18.7 \pm 2.4$ & $4.7 \pm 1.5$ & $7.9 \pm 7.2$ & $441 \pm 8.1$ & $451 \pm 6.3$ \\
\hline$\Sigma \mathrm{BHC}$ & $113 \pm 16$ & $131 \pm 19$ & $47.2 \pm 15.6$ & $62.0 \pm 22.9$ & $1461 \pm 28$ & $1556 \pm 26$ \\
\hline Heptachlor & $266 \pm 7.7$ & $271 \pm 3.7$ & $194 \pm 5.3$ & $199 \pm 4.8$ & $217 \pm 9.1$ & $234 \pm 7.4$ \\
\hline $\begin{array}{l}\text { Heptachlor } \\
\text {-epoxide (B) }\end{array}$ & $16.3 \pm 3.3$ & $19.1 \pm 1.8$ & $10.8 \pm 7.2$ & $12.9 \pm 5.2$ & $207 \pm 3.1$ & $209 \pm 8.8$ \\
\hline Aldrin & $37.7 \pm 3.2$ & $40.6 \pm 3.5$ & $26.0 \pm 3.8$ & $29.5 \pm 4.3$ & $241 \pm 5.3$ & $260 \pm 9.3$ \\
\hline Dieldrin & $16.1 \pm 8.9$ & $19.9 \pm 2.4$ & $3.1 \pm 4.3$ & $4.8 \pm 2.4$ & $250 \pm 3.0$ & $277 \pm 6.5$ \\
\hline
\end{tabular}

Bull. Chem. Soc. Ethiop. 2015, 29(2) 


\begin{tabular}{|l|c|c|c|c|c|c|}
\hline Endrin & $48.0 \pm 2.7$ & $56.7 \pm 2.7$ & $15.2 \pm 7.3$ & $18.9 \pm 5.2$ & $368 \pm 2.9$ & $387 \pm 4.1$ \\
\hline Endrin aldehyde & $90.1 \pm 2.1$ & $95.7 \pm 4.4$ & $6.8 \pm 6.4$ & $6.9 \pm 1.6$ & $\mathrm{ND}$ & $\mathrm{ND}$ \\
\hline Endrin ketone & $245 \pm 5.7$ & $249 \pm 9.8$ & $57.8 \pm 8.2$ & $68.5 \pm 2.8$ & $\mathrm{ND}$ & $\mathrm{ND}$ \\
\hline Cis-Chlordane & $74.0 \pm 8.2$ & $83.9 \pm 8.1$ & $4.2 \pm 3.6$ & $6.9 \pm 3.2$ & $306 \pm 8.6$ & $399 \pm 4.6$ \\
\hline $\begin{array}{l}\text { Trans- } \\
\text { Chlordane }\end{array}$ & $29.7 \pm 4.7$ & $26.3 \pm 5.2$ & $6.7 \pm 9.6$ & $8.4 \pm 4.1$ & $160 \pm 6.4$ & $191 \pm 7.2$ \\
\hline Endosulfan 1 & $31.3 \pm 3.8$ & $37.9 \pm 4.3$ & $7.7 \pm 7.7$ & $8.0 \pm 6.4$ & $184 \pm 8.5$ & $194 \pm 5.2$ \\
\hline Endosulfan 11 & $47.9 \pm 7.3$ & $49.6 \pm 6.8$ & $4.2 \pm 3.0$ & $5.8 \pm 8.6$ & $133 \pm 3.7$ & $139 \pm 7.9$ \\
\hline $\begin{array}{l}\text { Endosulfan } \\
\text { sulphate }\end{array}$ & $134 \pm 2.3$ & $139 \pm 10.1$ & $15.7 \pm 2.1$ & $17.9 \pm 4.9$ & $\mathrm{ND}$ & $\mathrm{ND}$ \\
\hline Methoxychlor & $48.3 \pm 7.3$ & $56.3 \pm 5.4$ & $9.2 \pm 5.5$ & $11.6 \pm 7.1$ & $\mathrm{ND}$ & $\mathrm{ND}$ \\
\hline p,p'-DDE & $36.8 \pm 4.2$ & $34.5 \pm 7.3$ & $1.9 \pm 7.0$ & $2.9 \pm 3.4$ & $127 \pm 5.0$ & $136 \pm 8.3$ \\
\hline p,p'-DDD & $63.9 \pm 3.8$ & $68.5 \pm 6.8$ & $4.6 \pm 3.8$ & $5.0 \pm 4.2$ & $\mathrm{ND}$ & $\mathrm{ND}$ \\
\hline p,p'-DDT & $69.1 \pm 7.5$ & $76.4 \pm 5.2$ & $7.2 \pm 7.3$ & $6.8 \pm 7.2$ & $2316 \pm 7.3$ & $2111 \pm 12$ \\
\hline$\Sigma$ DDT & $170 \pm 16$ & $180 \pm 19.3$ & $13.7 \pm 18$ & $14.8 \pm 15$ & $2443 \pm 12$ & $2247 \pm 21$ \\
\hline$\Sigma$ OCPs & $1367 \pm 99$ & $1455 \pm 106$ & $422 \pm 108$ & $476 \pm 98$ & $5968 \pm 91$ & $6092 \pm 107$ \\
\hline
\end{tabular}

Table 8. Mean concentrations (ng/g) of organochlorines in organs of male and female Chrysichthys nigrodigitatus during the wet season in Lagos Lagoon.

\begin{tabular}{|c|c|c|c|c|c|c|}
\hline \multirow[b]{3}{*}{ OCPs } & \multicolumn{6}{|c|}{ Lagos Lagoon } \\
\hline & \multicolumn{2}{|c|}{ Liver } & \multicolumn{2}{|c|}{ Kidney } & \multicolumn{2}{|c|}{ Gill } \\
\hline & Male & Female & Male & Female & Male & Female \\
\hline Alpha-BHC & $45.9 \pm 7.4$ & $54.6 \pm 7.0$ & $24.9 \pm 4.2$ & $26.4 \pm 3.8$ & $260 \pm 32.2$ & $282 \pm 20$ \\
\hline Beta-BHC & $12.9 \pm 5.1$ & $13.4 \pm 3.3$ & $4.3 \pm 3.3$ & $4.7 \pm 2.2$ & $627 \pm 53$ & $639 \pm 82$ \\
\hline Lindane & $21.6 \pm 8.5$ & $30.3 \pm 2.9$ & $8.1 \pm 6.5$ & $9.5 \pm 3.1$ & $96.2 \pm 8.9$ & $106 \pm 20$ \\
\hline Delta-BHC & $12.4 \pm 9.5$ & $14.9 \pm 1.0$ & $6.2 \pm 2.7$ & $8.5 \pm 2.3$ & $413 \pm 5.1$ & $455 \pm 26$ \\
\hline$\Sigma \mathrm{BHC}$ & $92.7 \pm 30.5$ & $113 \pm 14.2$ & $43.5 \pm 17$ & $49.1 \pm 12$ & $1396 \pm 99$ & $1482 \pm 149$ \\
\hline Heptachlor & $250 \pm 12$ & $255 \pm 9.3$ & $175 \pm 3.4$ & $183 \pm 9.2$ & $203 \pm 24$ & $235 \pm 7.4$ \\
\hline $\begin{array}{l}\text { Heptachlor } \\
\text {-epoxide (B) }\end{array}$ & $12.5 \pm 5.7$ & $14.9 \pm 4.1$ & $8.3 \pm 6.5$ & $11.3 \pm 3.4$ & $226 \pm 46$ & $211 \pm 9.3$ \\
\hline Aldrin & $33.1 \pm 4.3$ & $36.3 \pm 3.3$ & $28.6 \pm 2.1$ & $30.6 \pm 2.2$ & $228 \pm 4.7$ & $219 \pm 4.2$ \\
\hline Dieldrin & $13.6 \pm 3.5$ & $17.7 \pm 2.8$ & $3.9 \pm 1.2$ & $4.8 \pm 1.2$ & $240 \pm 5.8$ & $235 \pm 29$ \\
\hline Endrin & $44.4 \pm 9.3$ & $40.5 \pm 5.2$ & $13.1 \pm 5.4$ & $17.5 \pm 3.5$ & $319 \pm 45$ & $347 \pm 76$ \\
\hline Endrin aldehyde & $85.6 \pm 4.4$ & $84.2 \pm 3.4$ & $5.9 \pm 2.2$ & $8.4 \pm 4.1$ & ND & ND \\
\hline Endrin ketone & $229 \pm 6.1$ & $246 \pm 9.1$ & $52.6 \pm 2.8$ & $60.5 \pm 2.6$ & ND & ND \\
\hline Cis-Chlordane & $71.2 \pm 4.2$ & $72.8 \pm 2.3$ & $4.8 \pm 7.2$ & $4.4 \pm 2.3$ & $284 \pm 7.2$ & $310 \pm 56$ \\
\hline Trans-Chlordane & $25.9 \pm 3.6$ & $29.3 \pm 8.6$ & $5.5 \pm 3.4$ & $7.4 \pm 1.5$ & $140 \pm 8.1$ & $146 \pm 99$ \\
\hline Endosulfan 1 & $28.4 \pm 2.1$ & $32.8 \pm 12.6$ & $8.2 \pm 2.1$ & $9.3 \pm 4.4$ & $165 \pm 9.9$ & $217 \pm 8.5$ \\
\hline Endosulfan 11 & $43.0 \pm 7.9$ & $50.1 \pm 3.3$ & $3.2 \pm 1.1$ & $7.2 \pm 8.4$ & $119 \pm 5.3$ & $129 \pm 6.2$ \\
\hline Endosulfansulphate & $129 \pm 20.5$ & $135 \pm 6.2$ & $11.8 \pm 3.6$ & $15.9 \pm 16.3$ & ND & ND \\
\hline Methoxychlor & $43.1 \pm 2.4$ & $50.8 \pm 3.5$ & $9.6 \pm 2.3$ & $10.6 \pm 9.5$ & ND & ND \\
\hline $\mathrm{p}, \mathrm{p}^{\prime}-\mathrm{DDE}$ & $32.1 \pm 5.1$ & $36.5 \pm 6.1$ & $1.7 \pm 1.8$ & $1.8 \pm 2.3$ & $116 \pm 54.0$ & $105 \pm 5.9$ \\
\hline p,p'-DDD & $60.3 \pm 3.5$ & $64.8 \pm 2.2$ & $4.1 \pm 2.6$ & $5.2 \pm 5.4$ & ND & ND \\
\hline $\mathrm{p}, \mathrm{p}^{\prime}-\mathrm{DDT}$ & $61.1 \pm 6.3$ & $68.7 \pm 2.4$ & $9.7 \pm 3.7$ & $8.6 \pm 9.5$ & $1866 \pm 46$ & $1756 \pm 57$ \\
\hline$\Sigma$ DDT & $154 \pm 15$ & $170 \pm 11$ & $15.4 \pm 21$ & $15.6 \pm 17$ & $1981 \pm 100$ & $1862 \pm 93$ \\
\hline$\overline{\Sigma O C P s}$ & $1254 \pm 132$ & $1349 \pm 99$ & $389 \pm 81$ & $435 \pm 97$ & $5301 \pm 354$ & $5393 \pm 538$ \\
\hline
\end{tabular}

Table 9. Mean concentrations (ng/g) of organochlorines in the muscle tissues of male and female Liza grandisquamis during the dry and wet seasons.

\begin{tabular}{|l|c|c|c|c|c|c|c|c|}
\hline \multirow{3}{*}{ OCPs } & \multicolumn{4}{|c|}{ Agboyi Creek } & \multicolumn{4}{c|}{ Lagos Lagoon } \\
\cline { 2 - 9 } & \multicolumn{2}{|c|}{ Dry season } & \multicolumn{2}{c|}{ Wet season } & \multicolumn{2}{c|}{ Dry season } & \multicolumn{2}{c|}{ Wet season } \\
\cline { 2 - 9 } & Male & Female & Male & Female & Male & Female & Male & Female \\
\hline Alpha-BHC & $1.1 \pm 0.8$ & $1.7 \pm 0.7$ & $29.5 \pm 2.3$ & $8.9 \pm 2.1$ & $5.2 \pm 1.1$ & $53.9 \pm 5.2$ & $39.5 \pm 2.4$ & $0.6 \pm 0.4$ \\
\hline Beta-BHC & $0.4 \pm 0.3$ & $1.6 \pm 0.5$ & $148 \pm 8.4$ & $57.1 \pm 2.3$ & $1.0 \pm 0.3$ & $24.1 \pm 2.5$ & $31.4 \pm 1.2$ & $0.3 \pm 0.5$ \\
\hline Lindane & $0.6 \pm 0.4$ & $1.7 \pm 1.2$ & $63.4 \pm 2.2$ & $26.4 \pm 1.5$ & $14.0 \pm 2.4$ & $10.7 \pm 1.2$ & $71.4 \pm 3.8$ & $0.6 \pm 0.1$ \\
\hline
\end{tabular}

Bull. Chem. Soc. Ethiop. 2015, 29(2) 


\begin{tabular}{|c|c|c|c|c|c|c|c|c|}
\hline Delta-BHC & $0.7 \pm 0.8$ & $0.9 \pm 0.8$ & $23.9 \pm 1.3$ & $10.5 \pm 3.2$ & $3.7 \pm 2.2$ & $13.5 \pm 2.1$ & $21.4 \pm 2.5$ & ND \\
\hline$\Sigma \mathrm{BHC}$ & $2.8 \pm 2.3$ & $5.9 \pm 3.2$ & $265 \pm 14$ & $103 \pm 9.1$ & $23.9 \pm 6.0$ & $102 \pm 11.0$ & $164 \pm 9.9$ & $1.5 \pm 1.0$ \\
\hline Heptachlor & $0.9 \pm 0.2$ & $3.8 \pm 1.5$ & $55.9 \pm 3.3$ & $19.1 \pm 2.1$ & $27.7 \pm 3.4$ & $71.5 \pm 6.8$ & $14.4 \pm 3.5$ & $0.9 \pm 0.3$ \\
\hline $\begin{array}{l}\text { Heptachlor } \\
\text {-epoxide (B) }\end{array}$ & $1.1 \pm 1.2$ & $0.6 \pm 0.2$ & $44.1 \pm 2.4$ & $69.9 \pm 3.3$ & $17.9 \pm 2.5$ & $11.6 \pm 2.2$ & $24.5 \pm 1.5$ & ND \\
\hline Aldrin & $1.2 \pm 1.3$ & $1.2 \pm 0.6$ & $21.7 \pm 1.3$ & $12.0 \pm 2.1$ & $5.4 \pm 1.2$ & $26.1 \pm 1.4$ & $70.3 \pm 2.3$ & $1.2 \pm 0.2$ \\
\hline Dieldrin & $0.6 \pm 0.4$ & $0.6 \pm 0.3$ & $38.4 \pm 4.2$ & ND & $3.4 \pm 1.3$ & $22.7 \pm 2.2$ & $54.9 \pm 2.5$ & ND \\
\hline Endrin & $1.3 \pm 0.5$ & $0.5 \pm 0.9$ & $146 \pm 7.2$ & ND & $1.7 \pm 1.1$ & $117 \pm 6.4$ & $92.4 \pm 1.2$ & $0.5 \pm 0.2$ \\
\hline $\begin{array}{l}\text { Endrin } \\
\text { aldehyde }\end{array}$ & $1.2 \pm 0.8$ & $1.2 \pm 1.2$ & ND & ND & $2.3 \pm 1.3$ & ND & $609 \pm 8.4$ & ND \\
\hline Endrin ketone & $2.3 \pm 1.3$ & 0 & $458 \pm 8.5$ & ND & $22.4 \pm 2.3$ & $718 \pm 4.2$ & $274 \pm 5.5$ & ND \\
\hline $\begin{array}{l}\text { Cis- } \\
\text { Chlordane }\end{array}$ & $0.9 \pm 0.2$ & $0.8 \pm 0.2$ & $48.2 \pm 3.2$ & $94.1 \pm 3.3$ & $13.4 \pm 3.2$ & $77.6 \pm 2.5$ & $109 \pm 2.4$ & ND \\
\hline $\begin{array}{l}\text { Trans- } \\
\text { Chlordane }\end{array}$ & $0.7 \pm 0.3$ & $0.5 \pm 0.2$ & $114 \pm 2.3$ & ND & $5.1 \pm 1.2$ & $19.3 \pm 1.3$ & $39.5 \pm 3.4$ & $0.5 \pm 0.5$ \\
\hline Endosulfan 1 & $2.1 \pm 0.4$ & $1.1 \pm 1.2$ & $66.9 \pm 4.2$ & $127.1 \pm 6.4$ & $1.6 \pm 0.4$ & $21.3 \pm 3.2$ & $97.2 \pm 3.5$ & $0.7 \pm 0.3$ \\
\hline Endosulfan 11 & $1.2 \pm 0.1$ & $0.7 \pm 0.1$ & $88.3 \pm 3.4$ & ND & $2.5 \pm 0.6$ & $108 \pm 7.1$ & $124 \pm 7.1$ & ND \\
\hline $\begin{array}{l}\text { Endosulfan } \\
\text { sulphate }\end{array}$ & $0.9 \pm 1.4$ & $0.4 \pm 0.3$ & $340 \pm 8.1$ & ND & $12.4 \pm 2.2$ & ND & $91.5 \pm 3.3$ & ND \\
\hline Methoxychlor & 0 & 0 & $61.1 \pm 2.1$ & ND & $2.9 \pm 1.4$ & $25.5 \pm 3.7$ & $86.0 \pm 2.3$ & ND \\
\hline $\mathrm{p}, \mathrm{p}^{\prime}-\mathrm{DDE}$ & $0.8 \pm 0.3$ & $0.8 \pm 0.2$ & $44.8 \pm 1.3$ & ND & $1.9 \pm 0.2$ & $26.8 \pm 2.4$ & $37.7 \pm 3.2$ & ND \\
\hline $\mathrm{p}, \mathrm{p}^{\prime}-\mathrm{DDD}$ & $1.7 \pm 1.1$ & $2.1 \pm 1.2$ & $56.2 \pm 0.2$ & ND & $2.9 \pm 0.2$ & ND & $52.7 \pm 1.2$ & ND \\
\hline $\mathrm{p}, \mathrm{p}^{\prime}-\mathrm{DDT}$ & $0.9 \pm 0.3$ & $0.7 \pm 1.1$ & $195 \pm 3.1$ & ND & $7.5 \pm 2.4$ & $394 \pm 4.4$ & $141 \pm 3.1$ & ND \\
\hline$\Sigma$ DDT & $3.4 \pm 1.7$ & $3.6 \pm 2.5$ & $296 \pm 4.6$ & ND & $12.3 \pm 2.8$ & $420 \pm 6.8$ & $232 \pm 7.5$ & ND \\
\hline$\Sigma \mathrm{OCPs}$ & $20.4 \pm 12.1$ & $20.7 \pm 12.4$ & $2043 \pm 69$ & $425 \pm 26$ & $155 \pm 31$ & $1741 \pm 59$ & $2082 \pm 64$ & $5.2 \pm 2.5$ \\
\hline
\end{tabular}

Table 10. Mean concentrations ( $\mathrm{ng} / \mathrm{g}$ ) of organochlorines in gills of male and female Liza grandisquamis during the dry and wet seasons in Agboyi Creek.

\begin{tabular}{|c|c|c|c|c|}
\hline \multirow[b]{3}{*}{ OCPs } & \multicolumn{4}{|c|}{ Agboyi Creek } \\
\hline & \multicolumn{2}{|c|}{ Dry Season } & \multicolumn{2}{|c|}{ Wet Season } \\
\hline & Male & Female & Male & Female \\
\hline Alpha-BHC & $2.6 \pm 2.5$ & $5.1 \pm 4.4$ & $0.7 \pm 0.4$ & $4.8 \pm 4.0$ \\
\hline Beta-BHC & $1.4 \pm 2.2$ & $16.5 \pm 9.4$ & $0.6 \pm 0.1$ & $12.3 \pm 5.7$ \\
\hline Lindane & $2.9 \pm 2.8$ & $14.8 \pm 8.1$ & $0.6 \pm 0.2$ & $8.2 \pm 6.2$ \\
\hline Delta-BHC & $2.5 \pm 3.6$ & $28.8 \pm 9.6$ & $0.6 \pm 0.4$ & $23.7 \pm 13.1$ \\
\hline$\Sigma \mathrm{BHC}$ & $9.3 \pm 11.2$ & $65.2 \pm 31.6$ & $2.6 \pm 1.0$ & $48.9 \pm 29.0$ \\
\hline Heptachlor & $2.9 \pm 3.6$ & $14.1 \pm 12.2$ & $1.9 \pm 0.4$ & $7.7 \pm 6.9$ \\
\hline $\begin{array}{l}\text { Heptachlor } \\
\text {-epoxide (B) }\end{array}$ & $9.6 \pm 9.4$ & $24.5 \pm 8.7$ & $7.2 \pm 0.1$ & $18.5 \pm 8.4$ \\
\hline Aldrin & $2.9 \pm 2.4$ & $8.5 \pm 4.6$ & $1.7 \pm 0.5$ & $6.5 \pm 4.6$ \\
\hline Dieldrin & $7.9 \pm 5.3$ & $14.9 \pm 9.2$ & $4.9 \pm 0.6$ & $12.2 \pm 15.3$ \\
\hline Endrin & $39.6 \pm 7.3$ & $72.7 \pm 8.3$ & $35.8 \pm 0.3$ & $57.7 \pm 8.0$ \\
\hline Endrin aldehyde & ND & ND & ND & $323 \pm 24$ \\
\hline Endrin ketone & ND & ND & ND & ND \\
\hline Cis-Chlordane & $6.4 \pm 6.4$ & $32.9 \pm 9.9$ & $6.4 \pm 0.5$ & $29.3 \pm 8.5$ \\
\hline Trans-Chlordane & ND & $8.6 \pm 6.5$ & ND & $6.8 \pm 6.2$ \\
\hline Endosulfan 1 & $17.1 \pm 7.0$ & $56.1 \pm 17.7$ & $12.3 \pm 0.3$ & $49.0 \pm 9.4$ \\
\hline Endosulfan 11 & $24.1 \pm 9.1$ & $37.8 \pm 20.4$ & $12.8 \pm 0.5$ & $38.1 \pm 4.1$ \\
\hline Endosulfan sulphate & ND & ND & ND & ND \\
\hline Methoxychlor & ND & ND & ND & ND \\
\hline $\mathrm{p}, \mathrm{p}^{\prime}-\mathrm{DDE}$ & ND & $38.8 \pm 8.6$ & ND & $34.3 \pm 12.6$ \\
\hline $\mathrm{p}, \mathrm{p}^{\prime}-\mathrm{DDD}$ & ND & ND & ND & ND \\
\hline $\mathrm{p}, \mathrm{p}^{\prime}-\mathrm{DDT}$ & ND & ND & ND & ND \\
\hline$\Sigma$ DDT & ND & $38.8 \pm 8.6$ & ND & $34.3 \pm 12.6$ \\
\hline$\Sigma \mathrm{OCPs}$ & $120 \pm 62$ & $374 \pm 138$ & $85.8 \pm 4.2$ & $632 \pm 137$ \\
\hline
\end{tabular}

Bull. Chem. Soc. Ethiop. 2015, 29(2) 
The bioaccumulation of organochlorines in fishes is attributable to their lipophilic nature. They gain entrance into fishes by ingestion, dermal absorption and respiration. Accumulation of contaminants in fish lipids can occur by diffusion from the water across the gills and by transfer from the gut into the body after consumption of contaminated food. When these organic pollutants are taken up by the fish, they bioaccumulate, biomagnify and remain in the fish until they are eventually consumed by man. The processes of bioaccumulation and biomagnification of persistent contaminants may be affected by the fish's physiology, age, trophic levels, habitat, structure of food web and contaminant physicochemical properties [23].

Table 11. Mean concentrations (ng/g) of organochlorines in organs of male and female Liza grandisquamis during the dry season in Lagos Lagoon.

\begin{tabular}{|c|c|c|c|c|c|c|c|c|c|c|}
\hline \multirow[b]{3}{*}{ OCPs } & \multicolumn{10}{|c|}{ Lagos Lagoon } \\
\hline & \multicolumn{2}{|c|}{ Liver } & \multicolumn{2}{|c|}{ Kidney } & \multicolumn{2}{|c|}{ gill } & \multicolumn{2}{|c|}{ Large intestine } & \multicolumn{2}{|c|}{ Small intestine } \\
\hline & Male & Female & Male & Female & Male & Female & \begin{tabular}{l|} 
Male \\
\end{tabular} & Female & Male & Female \\
\hline Alpha-BHC & $33.4 . \pm 9.6$ & $15.6 \pm 3.4$ & $6.2 \pm 3.5$ & $7.1 \pm 7.3$ & $1627 \pm 6.1$ & $836 \pm 6.2$ & $48.3 \pm 3.6$ & $60.8 \pm 4.4$ & $18.2 \pm 6.3$ & $25.2 \pm 2.5$ \\
\hline Beta-BHC & $77.4 \pm 6.8$ & $54.6 \pm 1.8$ & $50.3 \pm 2.6$ & $37.6 \pm 3.5$ & $879 \pm 5.9$ & $1764 \pm 7.8$ & $238 \pm 4.5$ & $255 \pm 5.6$ & $150 \pm 8.7$ & $178 \pm 4.8$ \\
\hline \begin{tabular}{|l} 
Lindane \\
\end{tabular} & $15.4 \pm 7.4$ & $12.5 \pm 2.8$ & $15.3 \pm 4.4$ & $26.4 \pm 6.3$ & $1118 \pm 5.2$ & $629 \pm 6.5$ & $56.9 \pm 5.3$ & $41.2 \pm 6.5$ & $39.4 \pm 3.1$ & $38.2 \pm 6.4$ \\
\hline Delta-BHC & $26.3 \pm 5.2$ & $24.4 \pm 1.7$ & $34.6 \pm 3.2$ & $34.7 \pm 3.8$ & $543 \pm 3.3$ & $313 \pm 8.4$ & $105 \pm 8.4$ & $157 \pm 8.6$ & $20.9 \pm 6.2$ & $50.2 \pm 8.3$ \\
\hline IBHC & $153 \pm 29$ & $107 \pm 9.7$ & $107 \pm 3.7$ & $104 \pm 21$ & $4164 \pm 21$ & $3541 \pm 29$ & $448 \pm 228$ & $514 \pm 25$ & $228 \pm 24$ & $291 \pm 22$ \\
\hline Heptachlor & $39.2 \pm 4.9$ & \begin{tabular}{|l|}
$51.3 \pm 2.3$ \\
\end{tabular} & $34.8 \pm 6.1$ & $17.5 \pm 2.7$ & $1090 \pm 7.8$ & $661 \pm 6.4$ & $91.3 \pm 2.2$ & $109 \pm 7.2$ & $34.5 \pm 2.3$ & $79.8 \pm 3.5$ \\
\hline $\begin{array}{l}\text { Heptachlor } \\
\text {-epoxide (B) }\end{array}$ & $68.7 \pm 7.2$ & $80.8 \pm 3.4$ & $45.2 \pm 3.3$ & $35.2 \pm 3.9$ & $342 \pm 5.8$ & $589 \pm 2.1$ & $61.2 \pm 3.7$ & $128 \pm 6.6$ & $42.3 \pm 6.3$ & $65.2 \pm 6.2$ \\
\hline Aldrin & $36.3 \pm 4.4$ & $22.4 \pm 3.5$ & $20.5 \pm 6.3$ & $15.5 \pm 2.5$ & $600 \pm 2.9$ & $520 \pm 5.3$ & $102 \pm 7.2$ & $164 \pm 8.6$ & $91.8 \pm 5.3$ & $23.2 \pm 5.6$ \\
\hline Dieldrin & $48.2 \pm 6.3$ & $92.7 \pm 1.5$ & ND & $25.2 \pm 3.3$ & $293 \pm 7.4$ & $638 \pm 6.7$ & $84.2 \pm 8.5$ & $133 \pm 5.1$ & $134 \pm 8.4$ & $81.2 \pm 6.1$ \\
\hline Endrin & $67.5 \pm 7.1$ & $161 \pm 2.2$ & $201 \pm 4.2$ & $55.7 \pm 6.2$ & $453 \pm 7.5$ & $220 \pm 3.3$ & $59.4 \pm 5.2$ & $109 \pm 4.7$ & $394 \pm 3.8$ & ND \\
\hline \begin{tabular}{|l|} 
Endrin \\
aldehyde
\end{tabular} & ND & $106 \pm 2.4$ & ND & ND & $3571 \pm 5.8$ & $2773 \pm 8.5$ & $562 \pm 3.2$ & $848 \pm 8.6$ & $1323 \pm 5.3$ & ND \\
\hline Endrin ketone & $3763 \pm 4.6$ & $989 \pm 8.5$ & $658 \pm 5.2$ & $464 \pm 8.4$ & ND & $1909 \pm 4.2$ & $864 \pm 5.3$ & \begin{tabular}{|l|}
$1166 \pm 7.6$ \\
\end{tabular} & ND & $1240 \pm 5.5$ \\
\hline \begin{tabular}{|l|} 
Cis- \\
Chlordane
\end{tabular} & $26.3 \pm 3.1$ & $68.5 \pm 3.2$ & $25.6 \pm 7.1$ & $39.8 \pm 3.3$ & $654 \pm 5.3$ & $753 \pm 2.3$ & $111 \pm 6.4$ & $86.0 \pm 4.4$ & $52.8 \pm 4.1$ & $149 \pm 6.3$ \\
\hline $\begin{array}{l}\text { Trans- } \\
\text { Chlordane }\end{array}$ & $53.6 \pm 7.3$ & $27.3 \pm 7.4$ & $67.2 \pm 2.4$ & $21.2 \pm 2.5$ & $1084 \pm 3.5$ & $301 \pm 7.6$ & $22.3 \pm 4.3$ & $77.1 \pm 3.4$ & $131 \pm 6.3$ & $28.2 \pm 8.1$ \\
\hline Endosulfan 1 & $68.3 \pm 4.2$ & $81.5 \pm 3.2$ & $38.4 \pm 4.3$ & $17.3 \pm 9.1$ & $856 \pm 7.4$ & $629 \pm 5.2$ & $40.6 \pm 1.1$ & $112 \pm 5.2$ & $37.2 \pm 5.2$ & $64.7 \pm 6.3$ \\
\hline Endosulfan 11 & $174 \pm 9.5$ & $65.4 \pm 2.1$ & $68.4 \pm 7.4$ & $27.2 \pm 5.3$ & $256 \pm 3.4$ & $589 \pm 8.3$ & $100 \pm 9.4$ & $40.9 \pm 6.5$ & ND & $297 \pm 7.2$ \\
\hline \begin{tabular}{|l|}
$\begin{array}{l}\text { Endosulfan } \\
\text { sulphate }\end{array}$ \\
\end{tabular} & ND & $245 \pm 6.4$ & ND & \begin{tabular}{|l|} 
ND \\
\end{tabular} & $1039 \pm 2.3$ & $422 \pm 7.5$ & ND & ND & $\mathrm{ND}$ & ND \\
\hline Methoxychlor & $67.9 \pm 7.3$ & $56.3 \pm 5.4$ & $325 \pm 5.2$ & $225 \pm 3.2$ & $380 \pm 8.3$ & $436 \pm 5.5$ & $311 \pm 7.4$ & $279 \pm 7.3$ & $924 \pm 6.2$ & $101 \pm 5.2$ \\
\hline p,p'-DDE & $34.5 \pm 4.4$ & $81.8 \pm 6.3$ & ND & $39.5 \pm 6.3$ & $431 \pm 4.4$ & $211 \pm 6.2$ & $24.9 \pm 4.6$ & $34.2 \pm 7.2$ & $139 \pm 5.3$ & ND \\
\hline $\mathrm{p}, \mathrm{p}^{\prime}-\mathrm{DDD}$ & $\mathrm{ND}$ & $178 \pm 5.5$ & $\mathrm{ND}$ & ND & $1261 \pm 8.3$ & $628 \pm 8.4$ & ND & \begin{tabular}{|l|}
$78.7 \pm 6.5$ \\
\end{tabular} & ND & ND \\
\hline $\mathrm{p}, \mathrm{p}^{\prime}$-DDT & ND & $120 \pm 2.2$ & $\mathrm{ND}$ & ND & $839 \pm 4.3$ & $572 \pm 9.1$ & ND & ND & ND & $\mathrm{ND}$ \\
\hline$\Sigma$ DDT & $34.7 \pm 4.4$ & $377 \pm 14$ & $\mathrm{ND}$ & $39.5 \pm 6.3$ & $2530 \pm 17$ & $1412 \pm 24$ & $24.9 \pm 4.6$ & \begin{tabular}{|l|l|}
$1139 \pm 14$ \\
\end{tabular} & $139 \pm 5.3$ & ND \\
\hline इOCPs & $4592 \pm 95$ & $2524 \pm 75$ & $1585 \pm 55$ & $1081 \pm 77$ & $17305 \pm 104$ & $15386 \pm 81$ & $2877 \pm 90$ & $3872 \pm 114$ & $3522 \pm 82$ & $2415 \pm 82$ \\
\hline
\end{tabular}

The distribution profile of the organochlorines in the muscles, gills, livers, kidneys, large and small intestines indicate that different tissues and organs of fishes have varied concentrations of residues. The order of accumulation in the fishes was largely gills $>$ livers $>$ large intestines $>$ small intestines $>$ kidneys $>$ muscles. Gills accumulated much organochlorines than other organs in the fishes analysed. The levels of endosulfan and endosulfan sulphate were particularly high in the gills, confirming earlier studies [24]. However, the residue levels in the gills were reduced during the wet season, especially at Agboyi Creek due to dilution effect. The levels of organochlorines were higher in liver than in muscle tissues, and this is consistent with a study carried out by Metcalfe et al. [25]. The difference in patterns of these contaminants in liver and muscle tissue may reflect differences in metabolism of contaminants, content and 
composition of lipids, or the degree of blood perfusion in the various tissues. Liver plays a major role in the distribution, detoxification or transformation of these xenobiotics and constitutes an important site of pathological effects induced by persistent organic pollutants [26]. Moreover, contaminants tend to concentrate in the liver, reflecting a short-term exposure to pollutants [27].

Table 12. Mean concentrations (ng/g) of organochlorines in organs of male and female Liza grandisquamis during the wet season in Lagos Lagoon.

\begin{tabular}{|c|c|c|c|c|c|c|c|c|c|c|}
\hline \multirow[t]{3}{*}{ OCPs } & \multicolumn{10}{|c|}{ Lagos Lagoon } \\
\hline & \multicolumn{2}{|c|}{ Liver } & \multicolumn{2}{|c|}{ Kidney } & \multicolumn{2}{|c|}{ Gill } & \multicolumn{2}{|c|}{ Large intestine } & \multicolumn{2}{|c|}{ Small intestine } \\
\hline & Male & \begin{tabular}{|l|} 
Female \\
\end{tabular} & Male & Female & Male & Female & Male & Female & Male & Female \\
\hline Alpha-BHC & $33.5 \pm 4.5$ & $15.8 \pm 5.2$ & $6.3 \pm 3.1$ & $7.0 \pm 2.2$ & $1626 \pm 5.3$ & $836 \pm 8.8$ & $48.2 \pm 5.2$ & $59.5 \pm 2.1$ & $18.7 \pm 2.4$ & $25.0 \pm 3.2$ \\
\hline Beta-BHC & $77.8 \pm 6.2$ & $53.2 \pm 6.3$ & $50.4 \pm 5.2$ & $37.3 \pm 8.4$ & $878 \pm 3.6$ & $1764 \pm 9.6$ & $237 \pm 3.9$ & $255 \pm 3.3$ & $149 \pm 8.5$ & $1770 \pm 4.2$ \\
\hline Lindane & $15.4 \pm 3.3$ & $11.1 \pm 3.1$ & $15.2 \pm 4.3$ & $26.7 \pm 5.3$ & $1117 \pm 4.3$ & $629 \pm 7.2$ & $57.0 \pm 2.5$ & $41.3 \pm 7.2$ & $39.5 \pm 6.3$ & $38.2 \pm 2.4$ \\
\hline Delta-BHC & $26.7 \pm 2.4$ & $24.3 \pm 3.8$ & $34.6 \pm 2.6$ & $34.3 \pm 2.1$ & $543 \pm 3.2$ & $313 \pm 2.3$ & $105 \pm 4.3$ & $158 \pm 9.6$ & $20.6 \pm 1.1$ & $50.5 \pm 3.3$ \\
\hline$\Sigma$ हBHC & $153 \pm 16$ & $106 \pm 18$ & $107 \pm 15$ & $104 \pm 18$ & $4165 \pm 16$ & $3541 \pm 28$ & $447 \pm 16$ & $535 \pm 22$ & $228 \pm 18$ & $2084 \pm 13$ \\
\hline Heptachlor & $39.9 \pm 3.2$ & $51.4 \pm 5.5$ & $34.3 \pm 8.7$ & $17.7 \pm 1.1$ & $1088 \pm 4.2$ & $662 \pm 6.4$ & $91.3 \pm 8.4$ & $110 \pm 4.6$ & $34.2 \pm 5.2$ & $79.5 \pm 8.4$ \\
\hline $\begin{array}{l}\text { Heptachlor } \\
\text {-epoxide (B) }\end{array}$ & $67.6 \pm 5.6$ & $80.2 \pm 8.3$ & $44.2 \pm 7.6$ & $35.2 \pm 3.5$ & $343 \pm 3.1$ & $588 \pm 2.5$ & $61.1 \pm 2.5$ & $128 \pm 3.4$ & $42.1 \pm 2.1$ & $65.4 \pm 5.2$ \\
\hline Aldrin & $36.5 \pm 2.3$ & $22.5 \pm 3.4$ & $19.8 \pm 3.3$ & $15.5 \pm 3.6$ & $599 \pm 7.3$ & $520 \pm 3.2$ & $102 \pm 7.4$ & $163 \pm 8.3$ & $91.6 \pm 8.5$ & $23.7 \pm 4.5$ \\
\hline Dieldrin & $47.4 \pm 6.4$ & $91.4 \pm 8.3$ & ND & $25.2 \pm 8.5$ & $294 \pm 5.6$ & $639 \pm 4.3$ & $84.6 \pm 8.3$ & $132 \pm 2.6$ & $135 \pm 9.3$ & $80.3 \pm 2.1$ \\
\hline Endrin & $67.5 \pm 2.2$ & $161 \pm 3.4$ & $201.2 \pm 9.2$ & $55.8 \pm 3.3$ & $452 \pm 6.5$ & $219 \pm 5.5$ & $59.4 \pm 3.2$ & $108 \pm 7.5$ & $395 \pm 2.4$ & ND \\
\hline \begin{tabular}{|l|} 
Endrin \\
aldehyde
\end{tabular} & ND & $105 \pm 7.8$ & ND & ND & $3570 \pm 8.4$ & $2772 \pm 7.8$ & $562 \pm 2.4$ & $848 \pm 2.7$ & $1323 \pm 3.6$ & ND \\
\hline Endrin ketone & $3762 \pm 4.5$ & $989 \pm 8.3$ & $657 \pm 5.4$ & $463 \pm 2.8$ & ND & $1909 \pm 8.4$ & $864 \pm 2.3$ & $1167 \pm 6.5$ & $\mathrm{ND}$ & $1239 \pm 7.3$ \\
\hline $\begin{array}{l}\text { Cis- } \\
\text { Chlordane }\end{array}$ & $26.4 \pm 6.2$ & $68.3 \pm 8.4$ & $25.9 \pm 4.3$ & $39.5 \pm 4.2$ & $653 \pm 5.3$ & $752 \pm 6.3$ & $109 \pm 2.6$ & $87.6 \pm 4.4$ & $52.8 \pm 2.3$ & $148 \pm 9.6$ \\
\hline $\begin{array}{l}\text { Trans- } \\
\text { Chlordane }\end{array}$ & $53.4 \pm 5.3$ & $27.6 \pm 3.3$ & $67.1 \pm 8.5$ & $21.3 \pm 2.1$ & $1085 \pm 7.8$ & $301 \pm 8.5$ & $22.8 \pm 6.2$ & $77.5 \pm 2.3$ & $129 \pm 4.4$ & $28.7 \pm 3.2$ \\
\hline Endosulfan 1 & $68.5 \pm 8.8$ & $81.2 \pm 6.5$ & $38.6 \pm 4.3$ & $17.5 \pm 4.3$ & $856 \pm 2.7$ & $630 \pm 3.4$ & $39.5 \pm 1.6$ & $110 \pm 1.6$ & $37.2 \pm 2.3$ & $64.7 \pm 2.5$ \\
\hline $\begin{array}{l}\text { Endosulfan } \\
11 \\
\end{array}$ & $174 \pm 9.6$ & $65.5 \pm 5.7$ & $68.2 \pm 4.9$ & $27.5 \pm 2.6$ & $256 \pm 4.3$ & $589 \pm 5.2$ & $101 \pm 6.4$ & $39.3 \pm 3.4$ & ND & $298 \pm 6.8$ \\
\hline $\begin{array}{l}\text { Endosulfan } \\
\text { sulphate }\end{array}$ & ND & $244 \pm 7.4$ & ND & ND & $1039 \pm 7.6$ & $422 \pm 5.8$ & ND & ND & ND & ND \\
\hline Methoxychlor & $67.2 \pm 3.3$ & $56.5 \pm 4.6$ & $326 \pm 4.3$ & $224 \pm 3.7$ & $379 \pm 8.4$ & $436 \pm 3.3$ & $311 \pm 4.6$ & $279 \pm 2.6$ & $924 \pm 2.4$ & $102 \pm 7.3$ \\
\hline $\mathrm{p}, \mathrm{p}^{\prime}-\mathrm{DDE}$ & $34.6 \pm 2.2$ & $81.9 \pm 9.4$ & ND & $39.2 \pm 6.2$ & $431 \pm 9.5$ & $211 \pm 8.6$ & $24.8 \pm 2.3$ & $34.2 \pm 2.5$ & $139 \pm 2.3$ & ND \\
\hline $\mathrm{p}, \mathrm{p}^{\prime}-\mathrm{DDD}$ & ND & $178 \pm 8.6$ & ND & ND & $1260 \pm 6.6$ & $629 \pm 9.4$ & ND & $78.2 \pm 2.3$ & ND & ND \\
\hline $\mathrm{p}, \mathrm{p}^{\prime}-\mathrm{DDT}$ & ND & $119 \pm 6.5$ & $\mathrm{ND}$ & ND & $839 \pm 5.4$ & $572 \pm 6.7$ & ND & ND & ND & ND \\
\hline$\Sigma$ DDT & $34.8 \pm 2.2$ & $377 \pm 25$ & $\mathrm{ND}$ & $39.2 \pm 6.2$ & $2530 \pm 21$ & $1411 \pm 25$ & $24.8 \pm 2.3$ & $112 \pm 4.8$ & $139 \pm 2.3$ & ND \\
\hline$\Sigma \mathrm{OCPs}$ & $4593 \pm 76$ & $2524 \pm 123$ & $1584 \pm 75$ & $1081 \pm 63$ & $17305 \pm 109$ & $15386 \pm 123$ & $2878 \pm 72$ & $3872 \pm 76$ & $3522 \pm 63$ & $2416 \pm 70$ \\
\hline
\end{tabular}

The dietary surveys conducted in 100 families indicated that the amount of fishes consumed ranged from 20 to $200 \mathrm{~g}$ /day, with a mean value of $40 \mathrm{~g} /$ day. The mean consumption of fish in this study compares with the dietary surveys conducted in China where the consumption of fish increased from $27.5 \mathrm{~g} /$ day in 1989 to $30.5 \mathrm{~g}$ /day in 1997 [28]. In a survey conducted in 325 families in Coimbatore city, India, Muralidharan et al. [29] also reported a fish consumption of $47 \mathrm{~g} /$ day. In this study, respondents were asked to give information about the amount of species of fish they consume because fish consumption represents an important pathway for exposure to organochlorines. Although the vital organs of fish such as gills, livers and kidneys are sensitive to persistent organic pollutant accumulation, muscle forms the major edible portion in a fish. Therefore, muscle tissue alone was used in determining the dietary intakes to human body. The estimated daily intakes (EDI) of organochlorines by humans are shown in Table 13. $\Sigma$ BHC, aldrin, $\Sigma$ endrin, $\Sigma$ chlordane, $\Sigma$ heptachlor and $\Sigma$ DDT were used in estimating the daily intakes. The estimated daily intakes of the pesticides were within the acceptable daily intakes. The 
appraisal of dietary intake was based on comparison of acceptable daily intakes established by the joint FAO/WHO expert committee, Health Canada and USEPA (Table 13). Levels of organochlorines in the fish species analysed were within the permissible limits [30-32], suggesting that the fishes were safe for consumption.

Table 13. Acceptable daily intake (ADI) and estimated daily intake (EDI) (ng/kg body weight/day) of organochlorines by humans.

\begin{tabular}{|l|c|c|c|c|c|}
\hline \multirow{2}{*}{ Pesticides } & \multicolumn{3}{|c|}{ ADI } & \multicolumn{2}{c|}{ EDI } \\
\cline { 2 - 6 } & FAO/WHO & Health Canada & USEPA $\left(\mathrm{R}_{\mathrm{f}} \mathrm{D}\right)$ & C. nigrodigitatus & L. grandisquamis \\
\hline BHC & 42000 & 18000 & 18000 & 76.6 & 151 \\
\hline Heptachlor & 5000 & - & - & 152 & 57.1 \\
\hline Aldrin & 7000 & - & - & 17.2 & 40.2 \\
\hline Dieldrin & - & - & - & 33.1 & 31.4 \\
\hline Endrin & 6000 & - & - & 207 & 557 \\
\hline Chlordane & - & 3000 & 30000 & 25.0 & 92.6 \\
\hline Endosulfan & - & - & - & 172 & 283 \\
\hline Methoxychlor & - & - & - & 179 & 49.1 \\
\hline DDT & 1200000 & 1200000 & 30000 & 446 & 240 \\
\hline
\end{tabular}

Source: [30 -32].

\section{CONCLUSION}

A total of twenty organochlorines were detected in the fish samples. The residue levels were higher in the Lagos Lagoon than in Agboyi Creek while a higher concentration of the residues was observed during the dry season. The female fishes accumulated higher organochlorines than the male fishes. The order of accumulation of organochlorines in the fishes was gills $>$ livers $>$ large intestines $>$ small intestines $>$ kidneys $>$ muscles. The relationship between the maturation stage of female fishes and their levels of bioaccumulation of organochlorine pesticides will be a subject for further research.

\section{REFERENCES}

1. Fisk, A.T.; Hobson, K.A.; Norstrom, R.J. Environ. Sci. Technol. 2001, 19, 1508.

2. Lanfranchi, A.L.; Menone, M.L.; Miglioranza, K.S.; Janiot, L.J.; Aizpun, J.E.; Moreno, V.J. Mar. Pollu. Bull. 2006, 52, 74.

3. Dearth, M.A.; Hites, R.A. Environ. Sci. Technol. 1991, 25, 1279.

4. Muir, D.G.; Norstom, R.J.; Simon, M. Environ. Sci. Technol. 1990, 22, 1071.

5. Schlummer, M.; Moser, G.; McLahlan, M. Toxicol Appl. Pharmacol. 1998, 152, 128.

6. Covacia, A.; Gheorgheb, A.; Voorspoelsa, S.; Maervoeta, J.; Redekere, E.S.; Blustc, R.; Schepensa, P. Environ. Int. 2005, 31, 367.

7. Forget, J.; Solomon, K.R.; Ritter, L. IPCS Report PCS/95.39; 2001.

8. Rissato, S.R.; Galhiane, M.S.; Ximenes, V.F.; de Andrade, R.M.B.; Talamoni, J.L.B.; Libânio, M.; de Almeida, M.V.; Apon, B.M.; Cavalari, A.A. Chemosphere 2006, 65, 1949.

9. Davies, D.L.; Bradlow, H.L. Sci. Am. 1995, 273, 166.

10. Cocco, P.; Blair, A.; Congia, P.; Saba, G.; Flore, C.; Ecca, M.R.; Palmas, C. Arch. Environ. Health 1997, 52, 299.

11. Sarkar, A.; Nagarajan, R.; Chaphadkar, S.; Pal, S.; Singbal, S.Y. Water Res. 1997, 31, 195.

12. Usman, B.I.; Amin, S.M.N.; Arshad, A.; Aminur Rahman, M. Asian J. Animal Veter. Adv. 2013, 8, 154.

13. Voight, R.H. Proc. Estonian Acad. Sci.: Biol. Ecol. 2003, 52, 305.

14. Busacker, G.P.; Adelman, I.R.; Goolish, E.M. Am. Fish. Soc. 1990, 363. 
15. US Environmental Protection Agency (US EPA) Method 3570, Revision C, US EPA: Washington, DC, USA; 2002; $\mathrm{p} 9$.

16. US Environmental Protection Agency (US EPA). Method 3630, Revision B. SW-846 Manual, US EPA: Washington, DC, USA; 1996.

17. Pandit, G.G.; Sahu, S.K.; Sadasivan, S. J. Environ. Monit. 2002, 4, 431.

18. International Programme on Chemical Safety (IPCS); 2006. Available at: http://www.who.int/ipcs/publications/jmpr/jmpr_pesticide/en/index.html. Available at: http://www.epa.gov/ost/fishadvice/volum2/index.httml.

19. Naso, B.; Perrone, D.; Ferrante, M.C.; Bilancione, M.; Lucisano, A. Sci. Total Environ. 2005, 343, 83.

20. Unyimadu, J.P.; Udochu, A. J. Agric. Biotechnol.: Environ. 2002, 4, 14.

21. Ize-Iyamu, O.K.; Asia, I.O.; Egwakhide, P.A. Int. J. Phys. Sci. 2007, 2, 237.

22. Adeyemi, D.; Ukpo, G.; Anyakora, C.; Unyimadu; J.P. Am. J. Environ. Sci. 2008, 4, 649.

23. McIntyre, J.K.; Beauchamp, D.A. Sci. Total Environ. 2007, 372, 571.

24. Ondarza, P.M.; Miglioranza, K.S.B.; Gonzalez, M.; Shimabukuro1, V.M.; Aizpún, J.E.; Moreno, V.J. J. Braz. Soc. Ecotoxicol. 2010, 5, 41.

25. Metcalfe, C.; Metcalfe, T.; Ray, S.; Paterson, G.; Koenig, B. Mar. Environ. Res. 1999, 47, 1.

26. Evans, D.W.; Doodoo, D.K.; Hanson, P.J. Mar. Pollu. Bull. 1993, 26, 329.

27. Albaiges, J.; Farran, A.; Soler, M.; Gallifa, A.; Martin, P. Mar. Environ. Res. 1987, $22,1$.

28. Yang, N.; Matsuda, M.; Kawano, M.; Wakimito, T. Chemosphere 2006, 63, 1342.

29. Muralidharan, S.; Dhananjayan, V.; Jayanthi, P. Environ. Res. 2008, 109, 15.

30. Oostdan, J.V.; Gilman, A.; Dewailly, E. Sci. Total Environ. 1999, $230,1$.

31. FAO (2000). FAO/WHO. 2005. Vol. 2B: 61.

32. US Environmental Protection Agency (US EPA), Vol. 2, 3rd ed., 2006, 2-1-5-105. 